\title{
Improved Efficiency of an Adaptive Beam-Shaping Technique Based on Phase Gratings and Spatial Frequency Filtering
}

\author{
Yoshiki Nakata $^{*}$, Yuto Hirakawa ${ }^{1}$, Tsubasa Morizuka ${ }^{1}$, Yuki Kosaka ${ }^{1}$, Noriaki Miyanaga ${ }^{2}$ and Kazuhito Osawa ${ }^{3}$ \\ ${ }^{1}$ Institute of Laser Engineering, Osaka University, 2-6 Yamadaoka, Suita, Osaka 565-0871, Japan \\ ${ }^{2}$ Institute for Laser Technology, 1-8-4 Utsubo-honmachi, Nishiku, Osaka-city, Osaka 550-0004, \\ Japan \\ ${ }^{3}$ Murata Manufacturing Co., Ltd., 10-1, Higashikotari 1-chomme, Nagaokakyo, Kyoto 617-8555, \\ Japan \\ *Corresponding author's e-mail: nakata-y@ile.osaka-u.ac.jp
}

\begin{abstract}
Flattop beam shapes are in high demand for various laser applications, including ultra-high-power laser systems and surface processing, which includes interference laser processing and medical treatment. In this study, we report on the improvement of extraction efficiency in a beam shaping method using phase gratings and spatial frequency filtering. By organising the phase grating vector $k_{g}$ unparallel to the normal vectors $k_{n}$ of the sides of an aimed polygonal beam shapes, which are square and hexagonal in this study, the high-spatial frequency component is maintained in the spatial frequency filtering process, and high-quality beam shaping with good flatness and edge steepness can be obtained. In addition, the extraction efficiency of polygon-shaped beams from a Gaussian beam or flattop circular beam is discussed.
\end{abstract}

DOI: $10.2961 /$ jlmn.2020.03.2013

Keywords: beam shaping, efficiency, spatial light modulator, phase grating, flattop beam, polygonal beam

\section{Introduction}

Beam shape is one of the most important parameters in all laser applications. There is a strong need for lasers with a flat intensity distribution for a variety of applications, including ultra-high-power laser systems, such as the LFEX (Laser for Fast Ignition Experiment) at Osaka University, and area processing by lasers. In the former case, the flatness limits the maximum power because the laser glasses and optics can be damaged at a peak region [1-3]. In the latter case, a flattop beam shape is required for scribing [4], repairing [5], skin therapy [6] and interference pattern processing [714]. Our group has been studying area processing, including interference pattern machining, since the beginning of the $21^{\text {st }}$ century.

Figure 1 shows an example of interference laser processing. Figure 1(a) gives a schematic of laser processing by an interference pattern of four correlating beams, where spots align in a square matrix, as shown in Fig. 1(b). In this scheme, periodically aligned nanostructures can be fabricated by processing metal films using an ultrashort laser interference pattern, as shown in Fig. 1(c). In this case, the period of the interference pattern was $2.2 \mu \mathrm{m}$ and a 40.9-nmthick silver thin film on a sapphire (0001) substrate was processed by a single shot [15]. Here, the nanostructures are found to change with fluence and a flat beam is needed to fabricate uniform devices. In addition, tiling of the step scanning processing is required to fabricate wide and loss-less devices, as shown in Fig. 2. Considering these factors, we have investigated beam shaping methods that enable us to obtain flattop and rectangular beam shapes [16-18]. For the original beam shape, we have considered circular beam shapes, such as Gaussian, which are common in commercial lasers.
Various methods of beam shaping have been developed [19-24], but these methods have difficulties in shaping quality and wavefront accuracy. Alternatively, a method using phase grating and spatial frequency filtering in $\boldsymbol{4} \boldsymbol{f}$ systems has been developed $[18,25]$. This method is capable of beam and wavefront shaping simultaneously. As described in the next section, the lack of precision in beam shaping due to the limited spatial frequency is a problem. We have succeeded in improving the accuracy of the beam shaping by optimising the spatial frequency distribution $[16,17]$. In this study, we present the results of a typical beam shaping operation using our method and a discussion of the energy utilisation efficiency of the beam shaping operation.
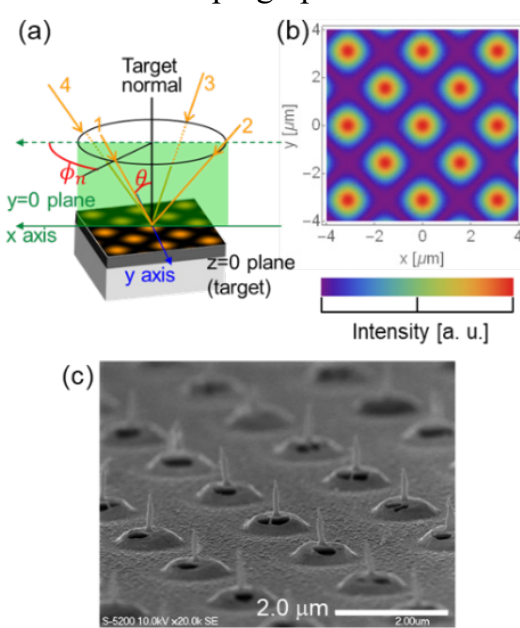

Fig. 1 (a) Schematic of interference laser processing. (b) Simulation of interference pattern. (c) Silver nanowhisker matrix fabricated by interference femtosecond laser processing. 

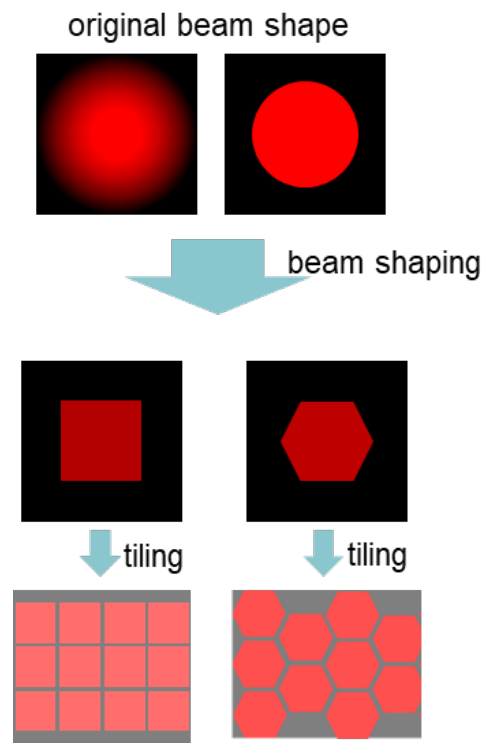

Fig. 2 Schematic of entire process from beam shaping to tiling.

\section{Scheme of beam shaping}

The scheme of adaptive beam shaping is illustrated in Fig. 3. A phase grating is encoded on a phase-only spatiallight modulator (SLM) put at a $4 f$ system and the phase fluctuates in between $\phi_{1}$ and $\phi_{2}$, as illustrated in the upper left inset. The phase distribution is added to the beam and first order diffracted beams are cut at the Fourier plane by the spatial frequency filter (SFF). In this scheme, the amplitude at the output plane is expressed by the following equation $[16,25]$ :

$$
E^{\prime} \propto E_{0} \cos \left(\frac{\Delta \phi}{2}\right) \times \exp \left(j \frac{\phi_{1}+\phi_{2}}{2}\right)
$$

and the amplitude can be attenuated by the phase depth $\Delta \phi=\phi_{2}-\phi_{1}$. Now, by controlling local phase depth $\Delta \phi(x, y)$, a beam can be shaped to a target shape.

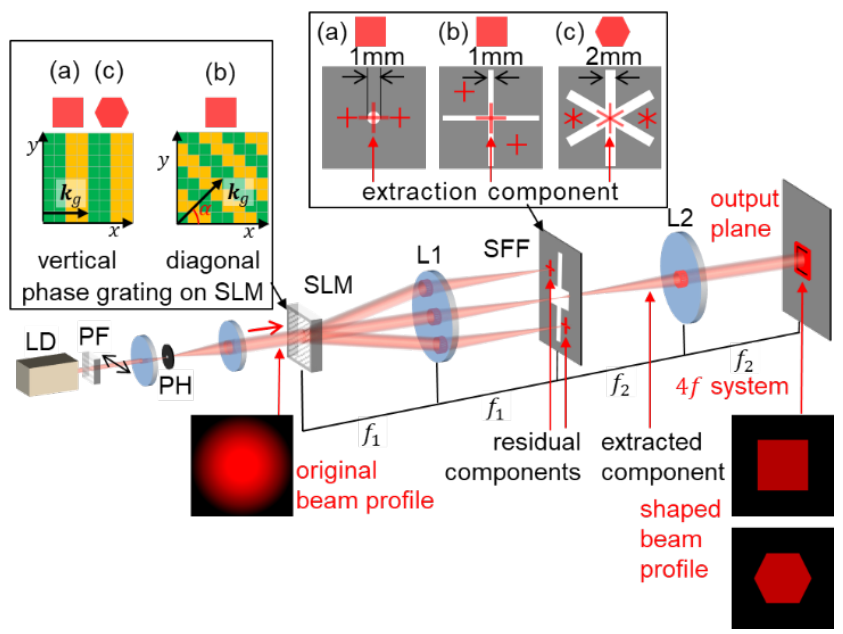

Fig. 3 Schematic of adaptive beam shaping. The same alphabetic numbers in the upper and the upper left illustrations correspond to each other. A rectangle is formed with (a) a vertical phase grating and (b) a diagonal phase grating. (c) a hexagon is formed with vertical phase gratings.
As shown in the upper left inset of Fig. 3, the phase grating can be vertical or diagonal. If the phase grating vector $k_{g}$ is parallel to the normal vectors of the sides, the residual component, which should be cut, overlaps with the extracted component. On this basis, the results of a beam shaping will vary with the vertical and diagonal phase gratings, as described in the next section.

Figure 4 illustrates an image of the extraction of polygonal beams from a Gaussian beam. Various shapes can be extracted from the Gaussian beam, but there will be losses. In later sections, we also discuss the extraction efficiency.

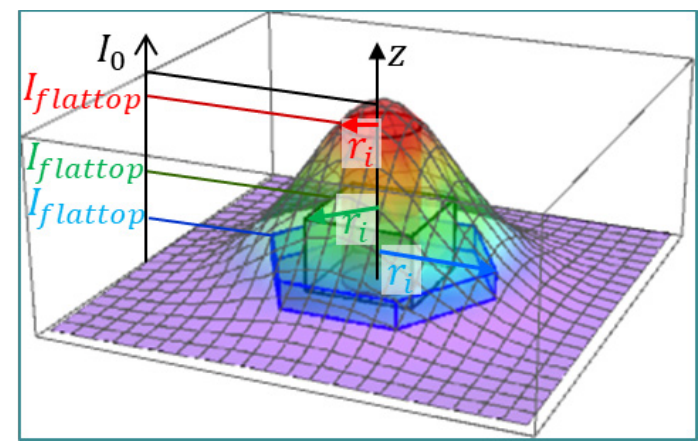

Fig. 4 Extraction of polygonal beams from a Gaussian

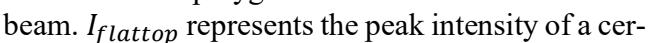
tain flattop beam. $r_{i}$ is the radius of a circle in which a regular polygon inscribes.

\section{Experimental results}

In this section, shaping to a flattop square and hexagon is shown. A laser diode at $780 \mathrm{~nm}$ with a Gaussian beam profile $(\Phi=9.8 \mathrm{~mm})$ was used. The phase grating overlapped the beam via an SLM (LCOS-SLM X-10468-02, Hamamatsu Photonics K. K.). The period of the vertical phase grating was $80 \mu \mathrm{m}$ (4 pixels), and that of the diagonal phase grating was $56.6 \mu \mathrm{m}(2 \sqrt{2}$ pixels $)$. The focal lengths of the lenses are $f_{1}=300 \mathrm{~mm}$ and $f_{2}=150 \mathrm{~mm}$, respectively.

The relationship between the phase gratings and the beam shape is shown in the upper left illustration of Fig. 3. In addition, the positional relationship between extracted and residual components on the SFF is shown in the upper illustration. An extracted component is located in the middle of each one and a phase grating diffracts the residual components. The shape of the components is like a cross in the case of a square beam and an asterisk in the case of a hexagonal beam. The shape of the SFF is such that in (a) a part of the extracted component is blocked, while in (b) and (c), the whole extracted components pass through and the residual components are blocked. On this basis, we investigated the change in beam shape due to efficient extraction of extracted components.

Here, we explain how a phase grating is created. First, the original beam profile is measured and a transfer function image is created by mapping the attenuation rate to the target beam profile. We then fit the attenuation curve $(I-\Delta \phi$ curve) to it to create a phase grating. The details are explained in Ref. [16].

Figure 5(a) shows the result of beam shaping to a flattop square by vertical phase grating. The diameter of the SFF was $1.0 \mathrm{~mm}$. The edge is not steep and a wavy structure can be seen on the flattop area. Now, the inverse Fourier 
transform image of the flattop square, which should be extracted through the SFF, is cross shaped. In this case, the residual components, which should be cut, overlap with it on the Fourier plane. As a result, the high-spatial frequency (HSF) component of the extracted component must be cut simultaneously by the SFF and the beam shaping quality was degraded. In contrast, they do not overlap on the Fourier plane in the case of the diagonal phase grating, as illustrated in Fig. 3(b) and extraction with the HSF component can be achieved using a cross-shaped SFF, with the quality far improved, as shown in Fig. 5(b). In this case, the edge increased in height within $20 \mu \mathrm{m}$ (from $20 \%$ to $80 \%$ in power). Based on the same concept, a beam can be shaped to a flattop hexagonal beam. The inverse Fourier transform image of the hexagonal beam is asterisk-shaped, as illustrated in the Fig. 3 (c). On the other hand, the residual and HSF components do not overlap on the Fourier plane in the case of vertical phase grating. As a result, good extraction with the HSF component can be achieved using an asterisk shaped SFF, and beam shaping to hexagon with good quality can be achieved. In this way, a preliminary result of the flattop hexagonal beam was obtained with good flatness and edge steepness, as shown in Fig. 5(c).

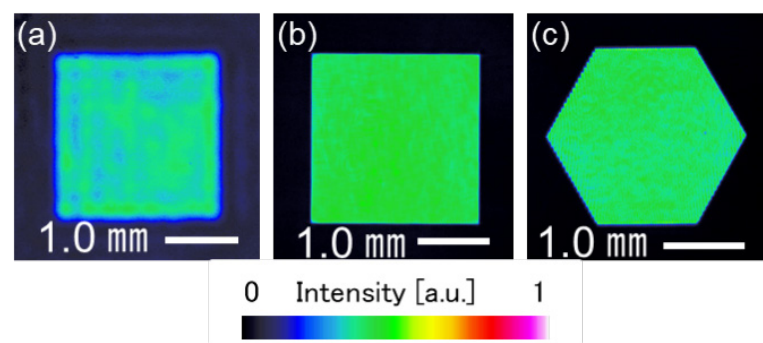

Fig. 5 Beam shaping to flattop square by (a) vertical and (b) diagonal phase grating. (c) Beam shaping to flattop hexagonal by vertical phase grating.

\section{Efficiency of extraction}

Our beam shaping technique is based on the extraction of a beam from an original beam, as illustrated in Fig. 4. Therefore, the efficiency of this method depends on the relationship between the original beam shape and the reshaped one. In this section, we assume that most commercial laser beam shapes are approximately Gaussian or flattop circular, and we consider the efficiency of extracting polygons from those beams.

\subsection{Efficiency of extraction from a Gaussian beam}

Many lasers have Gaussian beam shapes. In this section, we consider the efficiency of extracting a polygonal beam from a Gaussian beam. The ideal Gaussian beam is expressed by the following equation:

$$
I_{\text {Gaussian }}=I_{0} \exp \left(-\frac{2 r^{2}}{\omega_{0}^{2}}\right)
$$

where $r$ is the distance from the beam centre and $\omega_{0}$ is the radius, where the intensity is $I_{0} / e^{2}$. In addition, the area of a regular polygon inscribed in a circle of radius $r_{i}$ is

$$
S_{n}=\frac{1}{2} n r_{i}^{2} \sin \frac{2 \pi}{n}
$$

$$
\text { Efficiency }=\frac{I_{\text {flattop }} \times S_{n}}{\int_{r=0}^{\infty} I_{\text {Gaussian }}}
$$

where $n$ is the number of sides of the regular polygonal beam. Considering that the polygonal beam is inscribed in the Gaussian beam, as shown in Fig. 4, the efficiency for the ratio of the peak intensity of the Gaussian beam $I_{0}$ to that of the flattop polygonal beam $I_{\text {flat-top }}$ is shown in Fig. 6 .

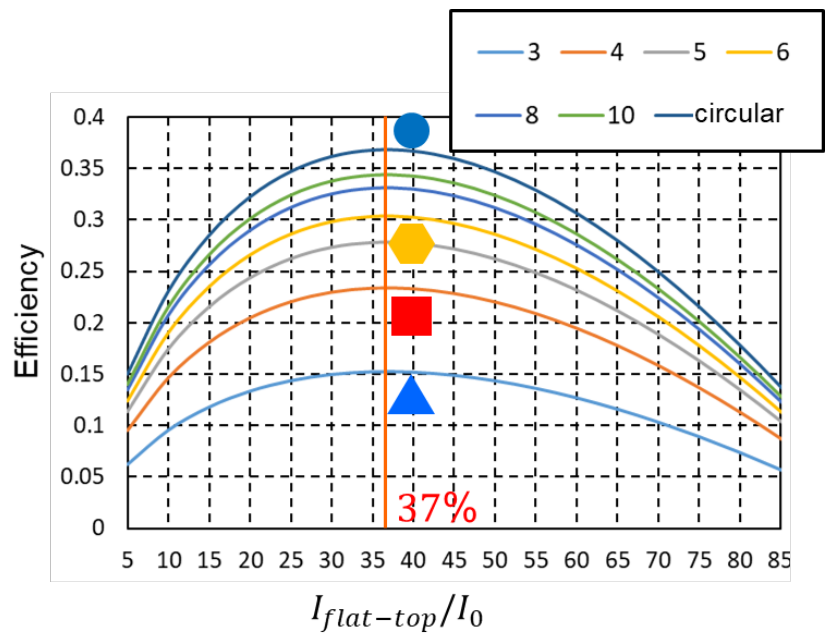

Fig. 6 Efficiency of extraction from a Gaussian beam.

In the case of all polygons, the efficiency is highest at $I_{\text {flat-top }} / I_{0}=0.37$. This is derived by substituting the solution of the equation:

$$
\frac{\mathrm{d} \text { Efficiency }}{\mathrm{d} r}=0 .
$$

, that is $r=\sqrt{1 / 2} \omega_{0}$, into the equation (4).

In the case of a flattop square beam, the maximum efficiency was $23 \%$. In contrast, it was increased to $30 \%$ in the case of a hexagonal beam. In the case of a flattop circular beam, it was $37 \%$. Considering that the long foot of a Gaussian beam results in a large loss, the use of Gaussian beams results in large losses in beam shaping compared to flattop circular beams.

\subsection{Efficiency of extraction from a flattop circular beam}

Some lasers have non-Gaussian beam profiles. For example, the beam shape of some Nd:YAG lasers is in between Gaussian and flattop, and excimer lasers have an approximately flattop beam. In this subsection, we consider the efficiency of extracting a polygonal beam from a flattop circular beam.

Figure 7 shows the efficiency of extracting regular polygonal beams $(3 \leq \mathrm{n} \leq 10)$ from a flattop circular beam, which is calculated by $S_{n} / \pi r_{i}{ }^{2}$. The ratio of polygons to flattop circular is $82.7 \%, 63.7 \%$ or $41.3 \%$ for a hexagon, square or triangle, respectively.

Here, there is no perfect Gaussian beam or flattop circular beam. In contrast, our beam shaping technique is adaptive to any beam shape. The efficiency changes to the shape of the original beam shape.

The efficiency is then expressed as follows: 


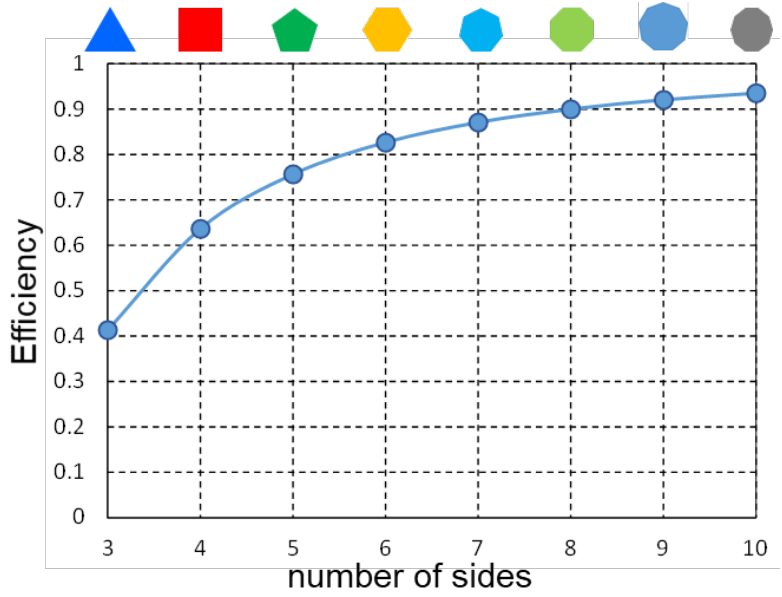

Fig. 7 Efficiency of extracting from a flattop circular beam.

\section{Summary}

We have demonstrated a beam shaping method that uses phase grating and spatial frequency filtering in a $4 f$ system. This new scheme, including the controlling of the direction of phase grating vector $k_{g}$, enables us to extract HSF component of an aimed beam shape. As a result, beam shaping to a flattop square or hexagonal beam with good flatness and edge steepness was demonstrated successfully. In addition, the efficiency of extraction from a Gaussian or flattop circular beam was discussed considering practical uses. When the original beam shape was Gaussian or flattop circular, the efficiency of the square shaping was $23 \%$ or $64 \%$, while the efficiency of the hexagonal shaping was $30 \%$ or $83 \%$. The advantages of this method could lead to expanded applications and new scientific discoveries using beam shaping.

\section{Acknowledgments}

This research was financially supported by the Japan Society for the Promotion of Science (JSPS) through a Grantin-Aid for Scientific Research (B) (No. 16H038850) and the Amada Foundation.

\section{References}

[1] N. Miyanaga, H. Azechi, T. Jitsuno, J. Kawanaka, Y. Fujimoto, H. Shiraga, K. Tsubakimoto, H. Kitamura, H. Matsuo, T. Sakamoto, Y. Izawa, K. Mima, K. A. Tanaka, R. Kodama, K. Kondo, H. Habara, and T. Kanabe: J. Phys. IV, 133, (2006) 1.

[2] S. Sakata, S. Lee, H. Morita, T. Johzaki, H. Sawada, Y. Iwasa, K. Matsuo, K. F. F. Law, A. Yao, M. Hata, A.

Sunahara, S. Kojima, Y. Abe, H. Kishimoto, A. Syuhada, T. Shiroto, A. Morace, A. Yogo, N. Iwata, M. Nakai, H. Sakagami, T. Ozaki, K. Yamanoi, T. Norimatsu, Y. Nakata, S. Tokita, N. Miyanaga, J. Kawanaka, H. Shiraga, K. Mima, H. Nishimura, M. Bailly-Grandvaux, J. J. Santos, H. Nagatomo, H. Azechi, R. Kodama, Y. Arikawa, Y. Sentoku, and S. Fujioka: Nat. Commun., 9, (2018) 3937. [3] S. W. Bahk, I. A. Begishev, and J. D. Zuegel: Opt. Commun., 333, (2014) 45.

[4] S. Rung, J. Barth, and R. Hellmann: Micromachines, 5, (2014) 943

[5] H. Y. Kim, W. S. Choi, S. Y. Ji, Y. G. Shin, J. W. Jeon, S. Ahn, and S. H. Cho: Appl. Phys. A Mater. Sci. Process., 124, (2018) 1.
[6] S. Thomsen, B. Baldwin, E. Chi, and J. Ellard: Proc. SPIE, 2970, (1997) 287.

[7] H. J. Gerritsen: J. Appl. Phys., 38, (1967) 2054.

[8] P. Simon and J. Ihlemann: Appl. Phys. a-Materials Sci. Process., 63, (1996) 505.

[9] Y. Nakata, T. Okada, and M. Maeda: Appl. Phys. Lett., 81, (2002) 4239.

[10] Y. Nakata, T. Okada, and M. Maeda: Jpn. J. Appl. Phys. Part 2 Lett, 42, (2003) L1452.

[11] A. Lasagni, C. Holzapfel, and F. Mücklich: Adv. Eng. Mater., 7, (2005) 487.

[12] Y. Nakata, N. Miyanaga, K. Momoo, and T. Hiromoto: Appl. Surf. Sci., 274, (2013) 27.

[13] S. Surdo and M. Duocastella: Adv. Sci., 6, (2019).

[14] Y. Nakata, M. Yoshida, and N. Miyanaga: Sci. Rep., $8,(2018) 13448$.

[15] Y. Nakata, N. Miyanaga, K. Momoo, and T.

Hiromoto: Jpn. J. Appl. Phys., 53, (2014) 096701.

[16] Y. Nakata, K. Osawa, and N. Miyanaga: Sci. Rep., 9, (2019) 4640.

[17] Y. Nakata, N. Miyanaga, and K. Osawa: Appl. Phys. A, 126, (2020) 1.

[18] K. Osawa, M. Yoshida, Y. Nakata, N. Miyanaga, A. Narazaki, T. Shoji, and Y. Tsuboi: Proc. SPIE, 10091, (2017) $100911 \mathrm{C}$.

[19] A. Laskin, V. Laskin, and A. Ostrun: Proc. SPIE, 9581, (2015) 93460R.

[20] J. A. Hoffnagle and C. M. Jefferson: Appl. Opt., 39, (2000) 5488.

[21] C. Han, Y. Ishii, and K. Murata: Appl. Opt., 22, (1983) 3644.

[22] T. Haist and W. Osten: J. Micro-Nanolithography MEMS MOEMS, 14, (2015) 041310.

[23] O. Homburg and T. Mitra: Laser Reson.

Microresonators, Beam Control XIV, 8236, (2012)

82360A.

[24] D. Nodop, J. Ruecker, S. Waechter, and M. Kahle: Opt. Lett., 44, (2019) 2169.

[25] V. Bagnoud and J. D. Zuegel: Opt. Lett., 29, (2004) 295.

(Received: July 16, 2020, Accepted: November 10, 2020) 